\title{
Microwave mediated impregnation of silver nanoparticles on silk fabric for hygienic clothing
}

\begin{abstract}
Textile materials and clothing are known to be susceptible to microbial attack, as these provide large surface area and absorb moisture required for microbial growth. Natural fibers have protein (keratin) and cellulose, etc., which provide basic requirements such as moisture, oxygen, nutrients and temperature for bacterial growth and multiplication. This often leads to objectionable odour, dermal infection, product deterioration, allergic responses and other related diseases. This necessitates the development of clothing that could provide a desired antimicrobial effect. Nanotechnology is an emerging, highly interdisciplinary field based on the ability to manipulate structural materials on the level of individual atom and molecules. Nanostructures are capable of enhancing physical properties of conventional textiles in areas such as antimicrobial properties, water repellence, soil resistance, antistatic, anti infrared and flame retardant properties, dye ability, colour fastness and strength of textile materials. Studies have been carried out in the present work to fine-tune the silver nanoparticles for special applications. Silver nanoparticles were biosynthesized in laboratory using Bacillus strain. Silk fabric was impregnated with silver nanoparticles using microwave irradiation method. Microwave irradiation is emerging as a rapid and green method of heating for nanoparticle synthesis and impregnation. The antibacterial activity of the impregnated fabrics was determined against 7 different gram $+v e$ and -ve bacterial strains. The results indicated that microwave impregnated silk fabric with silver nanoparticles had very good antibacterial activity (98.3\% inhibition). Bactericidal properties of the functionalized fabric do not change after washing. Production of such materials is extremely fast, convenient, and cost-effective.
\end{abstract}

Keywords: silver nanoparticles, antibacterial activity, silk fabric, microwave
Volume 5 Issue 4 - 2017

Smitha MS, Singh Rajni

Amity University Uttar Pradesh, India

Correspondence: Rajni Singh, Professor \& Additional Director, Amity Institute of Microbial Biotechnology, Amity University, Room no-307, J-3 Block, Sector I25, Noida-20 I303, UP, India, Tel +9। I204392900,

Email rsingh3@amity.edu, rajni_vishal@yahoo.com

Received: May 22, 2017 | Published: October 12, 2017

\section{Introduction}

Textile fiber provides an excellent environment for promoting the growth and multiplication of various microbes. ${ }^{1}$ The growth of the microbes on the fabrics results in a range of undesirable effects such as unpleasant odor, stains, reduction in strength of fabric and, moreover, affects the health of the wearer. These are main reasons why it is highly desirable to minimize the growth during their use and storage. All known natural and synthetic fibers can be used as base for preparation of antimicrobial materials.

The modification of the textiles surface using nanomaterials is becoming an interesting avenue for research. ${ }^{2}$ Among all metallic materials, silver is of particular interest because it is a powerful antibacterial agent, showing antimicrobial efficacy against bacteria, viruses, and microorganisms. This excellent antibacterial activity makes possible their use in different and varied fields such as food preservation, safe cosmetics, medical devices, water treatment, or textiles fabrics. ${ }^{3}$ The large surface area and high surface energy of nanomaterials ensures better immobilization of nanoparticles onto fabrics leading to an increase in durability of the textile functions. ${ }^{4}$ It also imparts other exciting properties especially antibacterial activity to the treated fabrics.

For example, Ti-nanoparticles on the textile materials are used in order to develop textile products with UV- protection and selfcleaning property. ${ }^{5}$ Silver nanoparticles are used as antimicrobial agent for wound dressing materials as well as for wound healing. Nano silver finishes have been applied on natural materials such as cotton, wool, and silk and also synthetic materials such as PET fabrics, with interesting antimicrobial results. ${ }^{6}$ The nano-silver particles are very popular antimicrobial agent due to an extremely large relative surface area, thus increasing their contact with bacteria and/or fungi and vastly improving their bactericidal and fungicidal effectiveness. They react with the sulfur-based proteins of the cell wall in microorganisms, inhibit with their metabolism, and thereby destroy them.

Antimicrobial finishing of textiles for biomedical purposes has become an important area of research and one of the fastest growing sectors of the textile market. The worldwide Antimicrobial Coatings Market was worth \$1.5billion in 2012 and is projected to reach $\$ 2.9$ billion by $2018 .{ }^{8}$ Shastri et al., ${ }^{9}$ has evaluated the antimicrobial activity of nanosilver-coated sock fabrics against foot pathogens. ${ }^{9}$ The need of the hour is developing simple methods to create safe biocidal fabrics with a long time of application. This direction is of great scientific and applied interest. This has necessitate the introduction of NPs in woven fabrics for clinical application. Impregnation of Ag NPs in wool, silk, cotton, nylon, viscose and polyamide have been done. Various methods like plasma or thermal sputtering, electrochemical way, laser ablation, and flaming synthesis have been identified for impregnating silver NPs onto various fabrics. ${ }^{10}$

In this paper, we report in situ fabrication of SNPs impregnated silk fiber by domestic microwave irradiation. The bactericidal efficacy of the SNDS (silver nanoparticles decorated silk) fibers was tested against both Gram positive and Gram negative bacteria. This study reveals that the SNDS fibers might be successfully employed for various applications. This is a cheap, portable, eco-friendly, and point of use system for creating hygienic textile. 


\section{Materials and methods}

\section{Biosynthesis of silver nanoparticles}

Lab isolated Bacillus strain was inoculated in nutrient broth and incubated at $37^{\circ} \mathrm{C}$ overnight. The culture was centrifuged and the pellet obtained was added with $1 \mathrm{M} \mathrm{AgNO}_{3}$ solution was in the ratio $1: 1$ and incubated at $37^{\circ} \mathrm{C}$. The reduction of silver ions to nanoparticles was characterized spectrophotometrically using UV-Vis spectrophotometer (Shimadzu model 1700 UV-Vis).

\section{Characterization of silver nanoparticles}

For structural and size characterization, HRTEM (Philips, CM10 model) was used with an operating voltage of $100 \mathrm{kV}$. The silver nanoparticles were dispersed on carbon-coated copper TEM grids for analysis.

\section{Microwave impregnation of silver nanoparticles on fabrics and antimicrobial activity}

For microwave assisted decoration of silk fiber by SNPs, they were placed in domestic microwave oven (Samsung model no MW73AD, $2450 \mathrm{MHz}, 800 \mathrm{~W})$ with rotating disk. The density of SNPs loaded on fiber and their sizes were varied with time (30-90s) and power (100-600W) of microwave irradiation. In order to avoid over heating/ burning of silk, the microwave was switched off after every $30 \mathrm{~s}$ of irradiation. In order to remove excess un-reacted silver precursor and loosely bound SNPs, treated silks were kept in warm water for $2 \mathrm{~h}$ followed by drying in hot air oven at $40^{\circ} \mathrm{C}$ for $8 \mathrm{~h}$.

To evaluate the antimicrobial activity of microwave irradiated impregnated silver nanoparticles on fabrics, Gram positive bacteria; Bacillus cereus (ATCC 14579), Bacillus subtilis (MTCC 6051), Bacillus licheniformis (ATCC 14580), Staphylococcus aureus (ATCC 25923), Streptococcus pyogenes (ATCC 19615) and Gram-negative bacteria: Escherichia coli (ATCC 11103), Klebsiella pneumoniae (ATCC 13883) cultures were inoculated in nutrient broth with silver nanoparticles impregnated fabrics. After overnight incubation at $37^{\circ} \mathrm{C}$, reduction in OD at $620 \mathrm{~nm}$ with reference to control indicated the inhibition of bacterial growth.

\section{Results}

\section{Biosynthesis of silver nanoparticles and its characterization}

The lab isolated Bacillus strain was able to synthesize silver nanoparticles at $37^{\circ} \mathrm{C}$. The UV-vis spectra showed strong SPR (showed strong surface plasmon resonance) band at $\sim 440 \mathrm{~nm}$ indicates the biosynthesis of silver NPs by the Bacillus strain (Figure 1). Studies have indicated that culture supernatants of some bacteria like Bacillus cereus, Bacillus subtilis, Bacillus licheniformis, Escherichia coli, Enterobacter cloacae, Klebsiella pneumonia, Lactobacillus acidophilus, Staphylococcus aureus, and Pseudomonas aeruginosa could induce the synthesis of silver nanoparticles. ${ }^{11-13}$ Studies using culture supernatants of bacteria like Pseudomonas proteolytica, Bacillus megaterium, Pseudomonas meridiana, Arthrobacter kerguelensis, Bacillus indicus, etc., were also proven its property to form extracellular nanoparticles very effectively. ${ }^{14}$

Formation of the silver nanoparticles was confirmed by HRTEM studies. The silver nanoparticles are well dispersed without aggregation. The representative HRTEM image showed welldispersed spherical-shaped silver nanoparticles ranging between 11 and 16nm (Figure 2).

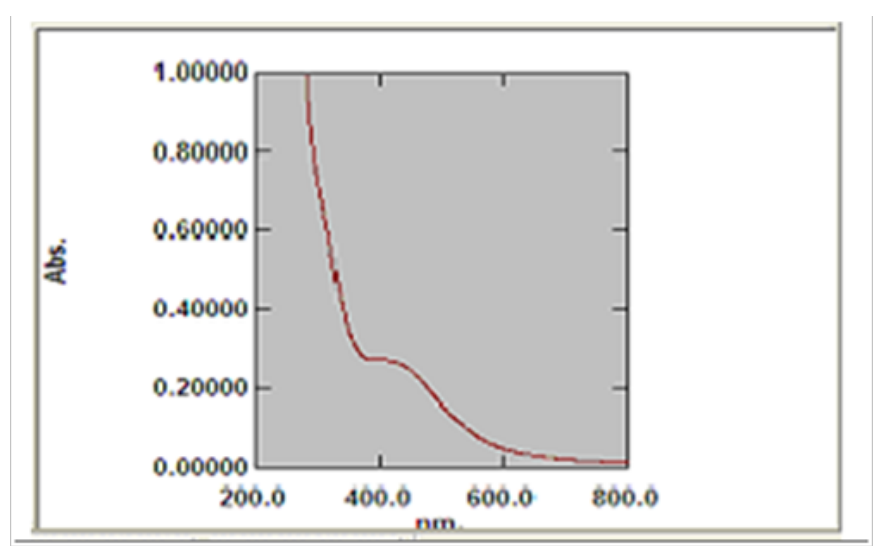

Figure I UV-Vis spectra of Bacillus sample of the reaction of aqueous solution of $\mathrm{AgNO}_{3}$ (IM) with pellet.

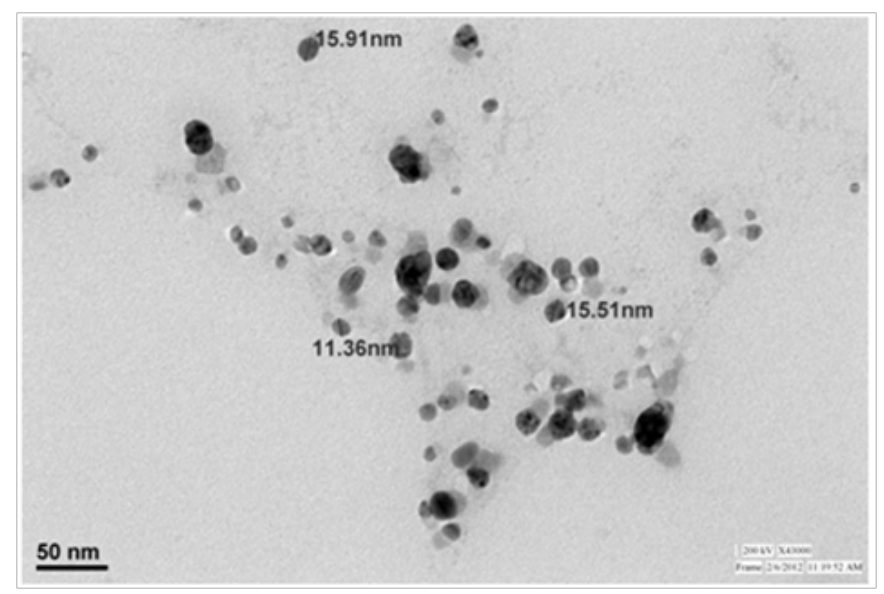

Figure 2 TEM of the synthesized silver nanoparticles.

Microwave heating is a transfer of electromagnetic energy to thermal energy with higher temperature homogeneity leading to accelerate the rate of reaction. Biological nanoparticles either through free amine group or cysteine residues or electrostatic attraction of negatively charged carboxylate groups present on the surface were responsible to provide good binding affinity between silk fibers and SNPs when irradiated with microwave.

\section{Antimicrobial activity of microwave impregnated silver nanoparticles}

The treated fabrics showed good antibacterial properties against both Gram-positive and Gram-negative bacteria. The results showed excellent antibacterial reduction ratio of the selected bacterial strains ranging from $66.7 \%$ to $98.3 \%$ for the fabrics treated. The antimicrobial efficacy has been observed even after 20 washes in the treated fabrics. Good antimicrobial activity has been observed against E. coli, Pseudomonas aeruginosa, Streptococcus, S. aureus and K. pneumoniae (Table 1). The flasks containing silver nanoparticles impregnated fabrics showed the inhibition against (i) Klebsiella pneumoniae and (ii) Bacillus subtilis after overnight incubation (Figure 3). The percent inhibition was found $98.3 \%$ and $75 \%$. Figure 4 is showing the bacterial growth inhibition by silver nanoparticles impregnated on silk fabrics with standard error. All the experiments were done in triplicates.

The antibacterial efficacy of SNPs extracted from Silver nanoparticles decorated cotton (SNDC) fibers was found to be more 
effective against Gram-negative bacteria than Gram-positive bacteria with MIC $38.5 \pm 0.93 \mu \mathrm{g} / \mathrm{mL}$ against Salmonella typhimurium MTCC98 and $125 \pm 2.12 \mu \mathrm{g} / \mathrm{mL}$ against Staphylococcus aureus MTCC-737 was also observed..$^{15}$ Textile materials with Ag NPs demonstrate high antibacterial activity, while fabrics doped with bimetallic composite $\mathrm{Ag} / \mathrm{Cu}$ have pronounced antimycotic properties. ${ }^{10}$

Table I Percentage inhibition of bacteria using microwave impregnated silver nanoparticles

\begin{tabular}{llll}
\hline Strains & Cfu/mL in control & Cfu/mL in samples treated with impregnated nanoparticles & Percent inhibition (\%) \\
\hline E coli & $11 \times 10^{7}$ & $36 \times 10^{6}$ & 66.7 \\
P. aeruginosa & $10 \times 10^{7}$ & $3.2 \times 10^{6}$ & 67.25 \\
Bacillus subtilis & $9 \times 10^{7}$ & $2.2 \times 10^{6}$ & 75.33 \\
Streptococcus & $10 \times 10^{7}$ & $4.8 \times 10^{6}$ & 95.2 \\
Staphylococcus aureus & $10 \times 10^{7}$ & $3.8 \times 10^{6}$ & 96.2 \\
Klebsiella pneumoniae & $9 \times 10^{7}$ & $1.5 \times 10^{6}$ & 98.3 \\
\hline
\end{tabular}

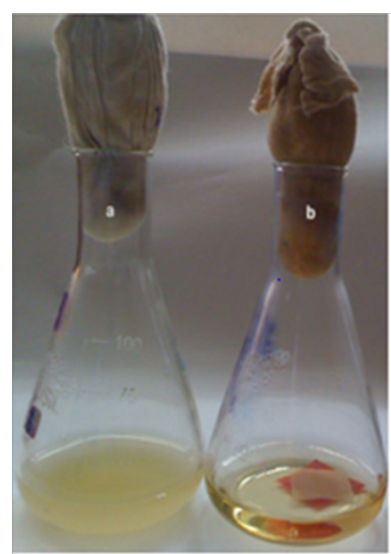

Figure 3(i)

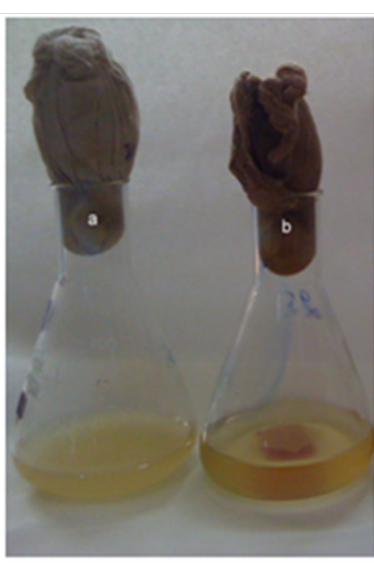

Figure 3 (ii)
Figure 3 Flasks containing silver nanoparticles impregnated fabrics showing inhibition against (i) Klebsiella pneumoniae and (ii) Bacillus subtilis after overnight incubation.

a. Positive Control.

b. Treated fabrics with impregnated silver nanoparticles.

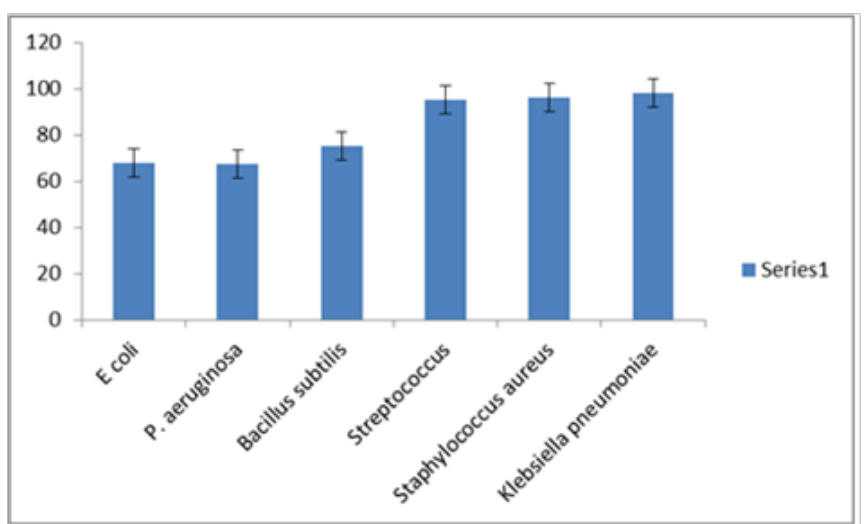

Figure 4 Bacterial growth inhibition by silver nanoparticles impregnated on silk febrics.

The antibacterial effect is greatly improved on account of the very large relative surface area, increasing their contact with bacteria. Nano silver has been found to be highly reactive with proteins. It therefore interferes with cell growth by seriously affecting cellular metabolism, through its contact with microbes. The foregoing discussions clearly point out that various types of nanomaterials applied to fabrics produce good antimicrobial effects, which are considered even better than antibiotic effects. The researches on the antimicrobial treatment of textiles clearly hold promise in medical applications in the near future. However, the impact of the different nanomaterials on the human health and environment needs to be well researched and established before they gain a commercial acceptance

\section{Conclusion}

Nano finishes applied onto fabrics have resulted in a number of desirable properties in the fabrics. Of these, the antimicrobial property has been one of the most promising functionality. Hence, it has been proved from the above results that the impregnation of microbial synthesized silver nanoparticles on fabrics paves a way for the production of hygienic clothes in textile industry to prevent from the nosocomial infections.

\section{Acknowledgements}

None.

\section{Conflict of interest}

Authors do not have any conflict of interest for the present manuscript.

\section{References}

1. Gao Y, Cranston R. Recent advances in antimicrobial treatments of textiles. Text Res J. 2008;78:60-72.

2. Rivero PJ, Urrutia A, Goicoechea J, et al. Nanomaterials for Functional Textiles and Fibers. Nanoscale Res Lett. 2015;10(1):501.

3. Firdhouse MJ, Lalitha P. Biosynthesis of Silver Nanoparticles and Its Applications. J Nanotechnol. 2015;2015:18.

4. Patra JK, Gouda S. Application of nanotechnology in textile engineering: an overview. J Eng Technol Res. 2013;5:104-111.

5. Sivakumar A, Murugan R, Sundaresan K, et al. UV protection and selfcleaning finish for cotton fabric using metal oxide nanoparticle. Ind $J$ Fiber Text Res. 2013;38:285-292.

6. Gokarneshan N, Gopalakrishnan PP, Jeyanthi B. Influence of Nanofinishes on the Antimicrobial Properties of Fabrics. ISRN Nano Materials. 2012;2012:8.

7. Prabhu S, Poulose EK. Silver nanoparticles: mechanism of antimicrobial action, synthesis, medical applications, and toxicity effects. Int Nano Lett. 2012;2:32. 
8. http://www.marketsandmarkets.com/PressReleases/antimicrobialcoatings.asp

9. Shastri JP, Rupani MG, Jain RL. Antimicrobial activity of nanosilvercoated socks fabrics against foot pathogens. J Text Ins. 2012;103:1-10.

10. Eremenko AM, Petrik IS, Smirnova NP, et al. Antibacterial and Antimycotic Activity of Cotton Fabrics, Impregnated with Silver and Binary Silver/Copper Nanoparticles. Nanoscale Res Lett. 2016;11:28.

11. Shahverdi AR, Minaeian S, Shahverdi HR, et al. Rapid synthesis of silver nanoparticles using culture supernatants of Enterobacteria: a novel biological approach. Process Biochem. 2007;42:919-923.

12. Kalimuthu K, Deepak V, Ramkumarpandian S, et al. Extracellular biosynthesis of silver nanoparticles by the culture supernatant of Bacillus licheniformis. Mater Lett. 2008;62:4411-4413.
13. Saifuddin N, Wong CW, Nur Yasumira AA. Rapid biosynthesis of silver nanoparticles using culture supernatant of bacteria with microwave irradiation. J Chem. 2009;6:61-70.

14. Shivaji S, Madhu S, Singh S. Extracellular synthesise of antibacterial silver nanoparticles using psychrophilic bacteria. Process Biochem. 2011;49:830-837.

15. Bhardwaj AK, Shukla A, Mishra RK, et al. Power and Time Dependent Microwave Assisted Fabrication of Silver Nanoparticles Decorated Cotton (SNDC) Fibers for Bacterial Decontamination. Front Microbiol. $2017 ; 8: 330$. 